\title{
A Retargetable Compilation Framework for Heterogeneous Reconfigurable Computing
}

\author{
ZAIN UL-ABDIN and BERTIL SVENSSON, Halmstad University
}

\begin{abstract}
The future trend in microprocessors for the more advanced embedded systems is focusing on massively parallel reconfigurable architectures, consisting of heterogeneous ensembles of hundreds of processing elements communicating over a reconfigurable interconnection network. However, the mastering of low-level microarchitectural details involved in the programming of such massively parallel platforms becomes too cumbersome, which limits their adoption in many applications. Thus, there is a dire need for an approach to produce high-performance scalable implementations that harness the computational resources of the emerging reconfigurable platforms.

This article addresses the grand challenge of accessibility of these diverse reconfigurable platforms by suggesting the use of a high-level language, occam-pi, and developing a complete design flow for building, compiling, and generating machine code for heterogeneous coarse-grained hardware. We have evaluated the approach by implementing complex industrial case studies and three common signal processing algorithms. The results of the implemented case studies suggest that the occam-pi language-based approach, because of its well-defined semantics for expressing concurrency and reconfigurability, simplifies the development of applications employing runtime reconfigurable devices. The associated compiler framework ensures portability as well as the performance benefits across heterogeneous platforms.
\end{abstract}

CCS Concepts: • Computer systems organization $\rightarrow$ Reconfigurable computing; Heterogeneous (hybrid) systems; $\bullet$ Software and its engineering $\rightarrow$ Retargetable compilers;

Additional Key Words and Phrases: Reconfigurable processor arrays, runtime reconfiguration, compiler frameworks, occam-pi

ACM Reference Format:

Zain Ul-Abdin and Bertil Svensson. 2016. A retargetable compilation framework for heterogeneous reconfigurable computing. ACM Trans. Reconfigurable Technol. Syst. 9, 4, Article 24 (August 2016), 22 pages.

DOI: http://dx.doi.org/10.1145/2843946

\section{INTRODUCTION AND MOTIVATION}

The computational requirements of high-performance embedded applications, such as baseband, video, and radar signal processing, have reached a level where they cannot be met with traditional digital signal processors. Massively parallel reconfigurable architectures offer the possibility to meet the growing computational demands of such applications with dynamically varying workloads. They are typically made up of hundreds of highly optimized heterogeneous functional blocks or even programcontrolled processing elements composed in an interconnection network that can be reconfigurable.

This research was supported in part by the CERES research program funded by the Knowledge Foundation, the ELLIIT strategic research initiative funded by the Swedish government, and ARTEMIS Joint Undertaking under grant agreement number 100230.

Authors' addresses: Z. Ul-Abdin and B. Svensson, CERES, School of Information Technology, Halmstad University, P.O. Box 823, SE-301 18 Halmstad, Sweden; emails: zain-ul-abdin@hh.se, bertil.svensson@hh.se. Permission to make digital or hard copies of all or part of this work for personal or classroom use is granted without fee provided that copies are not made or distributed for profit or commercial advantage and that copies bear this notice and the full citation on the first page. Copyrights for components of this work owned by others than the author(s) must be honored. Abstracting with credit is permitted. To copy otherwise, or republish, to post on servers or to redistribute to lists, requires prior specific permission and/or a fee. Request permissions from Permissions@acm.org.

2016 Copyright is held by the owner/author(s). Publication rights licensed to ACM.

ACM 1936-7406/2016/08-ART24 \$15.00

DOI: http://dx.doi.org/10.1145/2843946 
However, their widespread adoption is constrained due to the requirement of mastering microarchitectural details and using disparate low-level proprietary languages. The procedural models of high-level programming languages, such as $\mathrm{C}$, rely on sequential control flow, procedures, and recursion, which are difficult to adapt for reconfigurable arrays. Furthermore, because these languages were originally designed for sequential computers with unified memory systems, applying them for arrays of reconfigurable processing units with distributed memories results in inefficient use of available hardware, leading to increased power consumption and increased communication delays.

A new approach is direly needed and we propose one based on the concurrent programming model of occam-pi [Welch and Barnes 2005] and its associated compiler framework. The explicit expression of concurrency in occam-pi, with its ability to describe computations that reside in different memory spaces, together with the facility of expressing dynamic parallelism, dynamic process invocation mechanisms, and the language support for placement attributes, makes it suitable for mapping applications to a wide class of coarse-grained reconfigurable architectures. The framework presented in this article completes the design flow (methodology and tools) to compile the applications developed in the higher-level language to the selected architectures belonging to the category of reconfigurable arrays.

In earlier work, we have demonstrated the feasibility of using the occam-pi language to program industrial case studies onto emerging examples of heterogeneous reconfigurable architectures viz. XPP [Ul-Abdin and Svensson 2011], Ambric [Ul-Abdin et al. 2011; Ul-Abdin and Svensson 2012], and P2012 [Ul-Abdin et al. 2012; Gebrewahid et al. 2013]. The contributions of this article are as follows:

-We present a retargetable compilation tool chain for compiling and executing programs written in a high-level concurrent language that follows a minimalist approach.

-We describe how our approach can be applied to structure code generation in such a way that it facilitates code generator reuse when porting to a new hardware platform.

-We demonstrate the practical usefulness of the approach by implementing signal processing kernels on three different massively parallel reconfigurable architectures with varying degree of granularity, heterogeneity, and parallelism.

In the rest of the article, we first review some related work and present the three targeted reconfigurable architectures. We then provide an overview of the occam-pi language and describe the different phases of the compiler framework. Finally, the experimental results from the case studies are discussed.

\section{RELATED WORK}

\subsection{High-Level Programming Languages}

There has been a number of initiatives in both industry and academia to address the requirement of high-level languages for reconfigurable silicon devices. The most prominent among those used for reconfigurable computing are based on the CSP computation model. However, they differ from each other in the way they expose parallelism. For instance, while Handel-C [Han 2002] and Streams-C [Frigo et al. 2001] both have C-like syntax, Streams-C relies entirely on the compiler to expose parallelism, whereas Handel-C offers extensions. These extensions allow statement-level parallel constructs to identify a collection of instructions to be executed in parallel. The latter is similar to the approach taken in Mobius [Mob 2006]. All of these languages have been implemented for fine-grained architectures, while we are interested in targeting coarse-grained architectures. Another important feature lacking in the aforementioned languages, except Pebble [Luk and Mckeever 1998], is the ability to 
express runtime reconfiguration. With Pebble, the reconfigurablity support is provided at a very low level, describing individual logic blocks mainly intended for fine-grained architectures, whereas we are interested in exploring the abstractions to support reconfigurablity at the task and process level, which is more suitable for coarse-grained architectures. These limitations of the aforementioned languages have motivated us to suggest using the occam-pi language, which provides platform-independent abstractions and at the same time supports expression of reconfigurability that enables the programmer to target a variety of coarse-grained architectures. 0ccam-pi can also be adopted for fine-grained architectures and in that case it will closely resemble the Mobius language.

Apart from the previously mentioned language-based approaches, there have been attempts to exploit the dynamic reconfiguration capabilities of reconfigurable architectures by implementing a library of custom hardware modules each supporting a specific instruction. These custom instruction modules are reconfigured onto the FPGA under the control of a global controller that resides on the FPGA [Wirthlin and Hutchings 1995]. Burns et al. [1997] have proposed a similar approach in which the reconfiguration process is controlled by a runtime system that is executed on a host processor. Greaves and Singh [2008] have proposed a concurrent library called Kiwi to generate hardware through their associated synthesis framework from C\# parallel programs.

\subsection{Compilation Frameworks and Techniques}

The related work presented here covers some prominent compilers based on their relevance to the field of reconfigurable computing. We distinguish these efforts based on their support for input languages and target architectures. For a detailed review of compilers for reconfigurable computing systems, see Cardoso et al. [2010].

There are also frameworks such as the Dynamically Reconfigurable Embedded System Compiler (DRESC) [Mei et al. 2002] and the XPP vectorizing compiler (XPP-VC) [Cardoso and Weinhardt 2002] for coarse-grained architectures. All of these use C as the input language for application description and perform advanced program analysis to identify loops in the source code, which can then be transformed into pipelines. The loop-based transformations are limited to innermost loops that do not involve function calls. In contrast to the $\mathrm{C}$ language approach, the occam-pi language allows the programmer to explicitly describe the statements to be executed in parallel by using the PAR construct. Thus, our compiler framework does not require the loop-level transformations for extracting parallelism.

The RAW-StreamIt compiler [Gordon et al. 2002] developed at MIT was intended to compile a stream processing language called StreamIt to a scalable processor array, namely, Reconfigurable Architecture Workstation (RAW). The compiler produces stream graphs as an intermediate representation and generates parallel code for exploiting fine-grained parallelism.

The Polymorphic Pipeline Array proposed by Park et al. [2009] exploits the finegrained and coarse-grained parallelism in the compute-intensive kernels by the help of a virtualization controller embedded in each core. The compiler produces a single schedule that is then mapped dynamically by using the expanding approach to convert the schedule for multiple cores. Although the approach is effective in limiting the intercore communication, thereby reducing the buffer sizes, it needs dedicated virtualization controllers.

Another significant approach for code generation to coarse-grained reconfigurable architectures is presented in Lee et al. [2003], where the focus is on performing loop pipelining and partitioning onto a generic reconfigurable architecture template. Similarly, the code generator for Tightly Coupled Processor Arrays [Boppu et al. 2014] implements loop parallelization by separating control flow from the data flow and is 


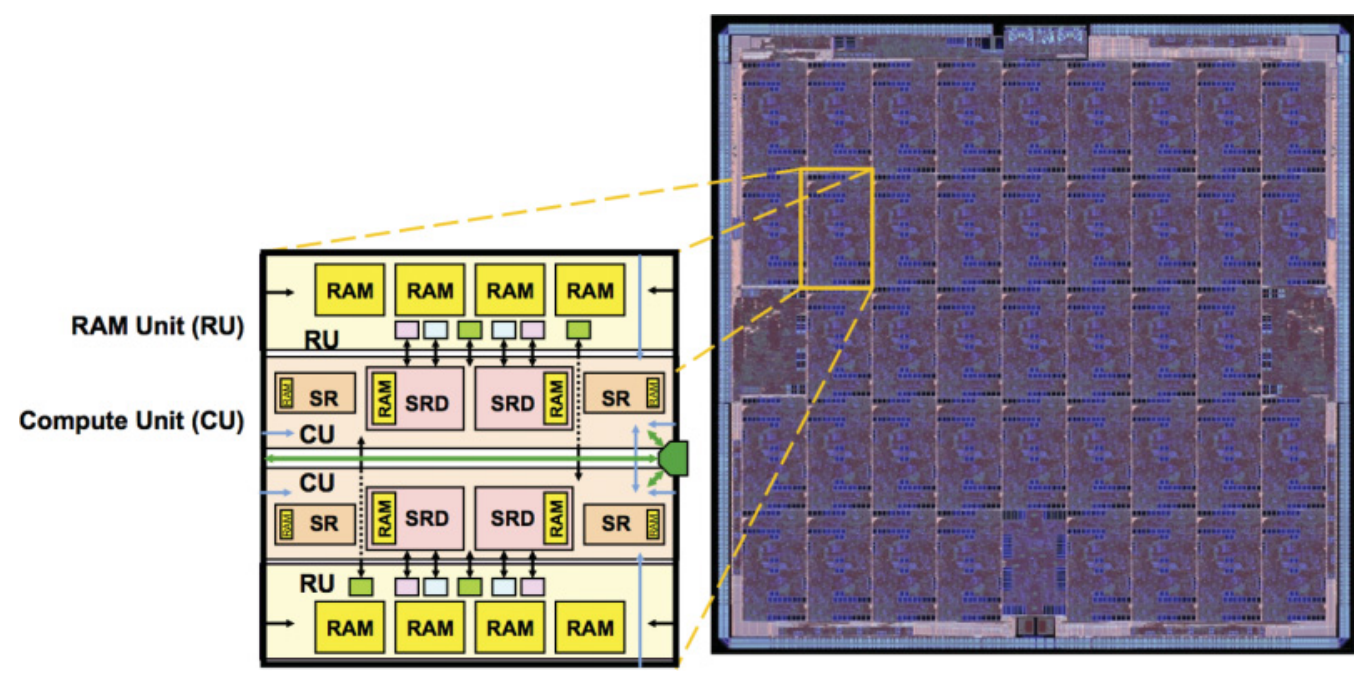

Fig. 1. Ambric architecture.

able to handle nested loops with a significant reduction in code size. Papakonstantinou et al. [2011] have targeted FPGAs by developing a high-level synthesis framework that transforms CUDA kernels into coarse-grained configurations.

A practical benefit of our methodology compared to the listed approaches lies in its seamless integration with the existing proprietary languages. This enables a high degree of compatibility and easy integration into and interoperability with existing software development environments.

\section{MASSIVELY PARALLEL RECONFIGURABLE ARRAYS}

This section provides an overview of the three massively parallel target architectures considered in our evaluation, as well as an outline of their proprietary programming techniques.

\subsection{Ambric}

Ambric (see Figure 1) is an asynchronous array of so-called brics. Each bric is composed of two pairs of Compute Units (CUs) and RAM Units (RUs) [Jones and Butts 2006]. The CU consists of two 32-bit Streaming RISC (SR) processors, two 32-bit Streaming RISC processors with DSP extensions (SRD), and a 32-bit reconfigurable channel interconnect. The RU consists of four banks of RAM along with a dynamic channel interconnect to facilitate communication with these memories. The Am2045 device has a total of 336 processors in 42 brics.

The individual software objects for Ambric are programmed in aJava or assembly language, which are then linked together using a proprietary language called aStruct. Thus, the programmer has to use two languages to implement an application on Ambric.

\subsection{PACT XPP}

The eXtreme Processing Platform (XPP) [Baumgarte et al. 2003] from PACT XPP is composed of ALU-PAEs (Processing Array Elements) that perform multiplication besides other common arithmetic and logic operations. The XPP64-A1 processor (see Figure 2) consists of an $8 \times 8$ array of ALU-PAEs, along with 16 RAM-PAEs. The forward register (FREG) and backward register (BREG) objects are mainly used for routing data and event packets, besides providing support for addition, subtraction, 


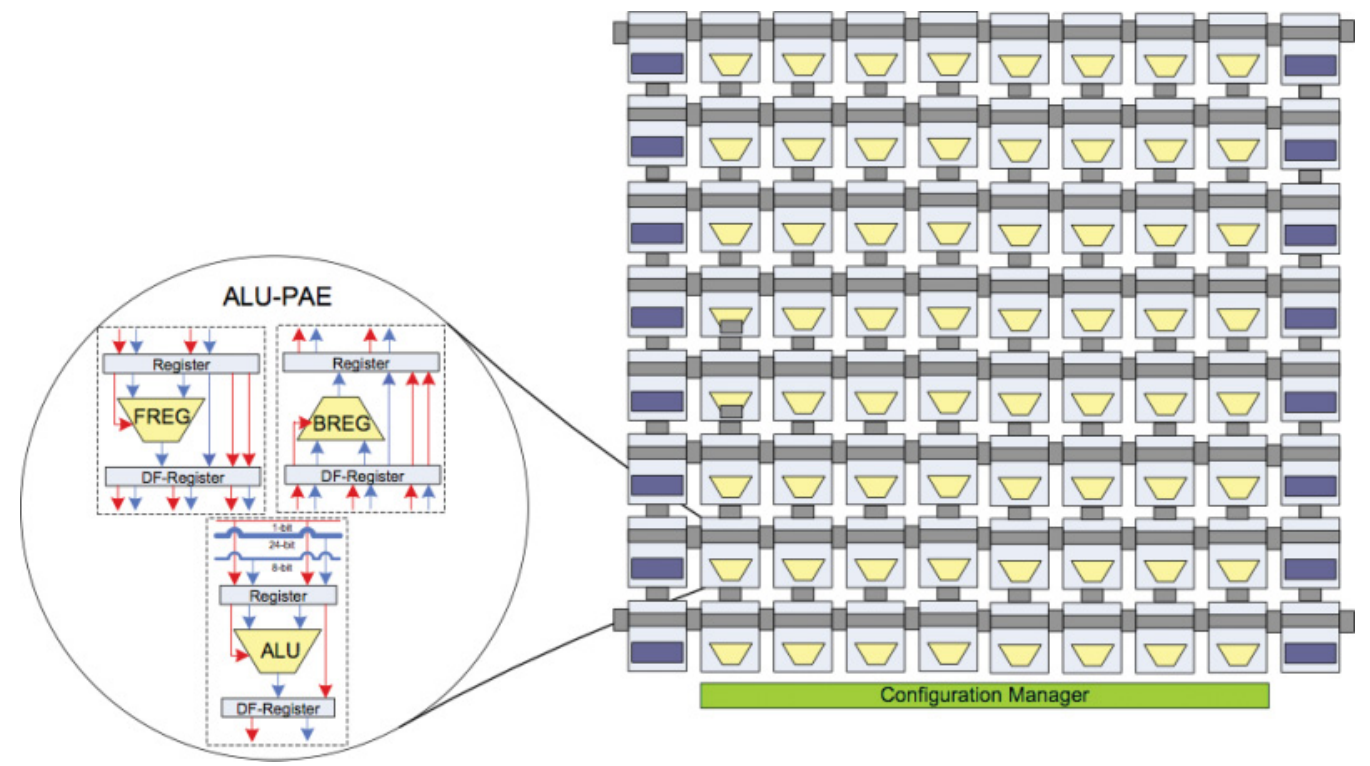

Fig. 2. Block diagram of XPP64-A1.

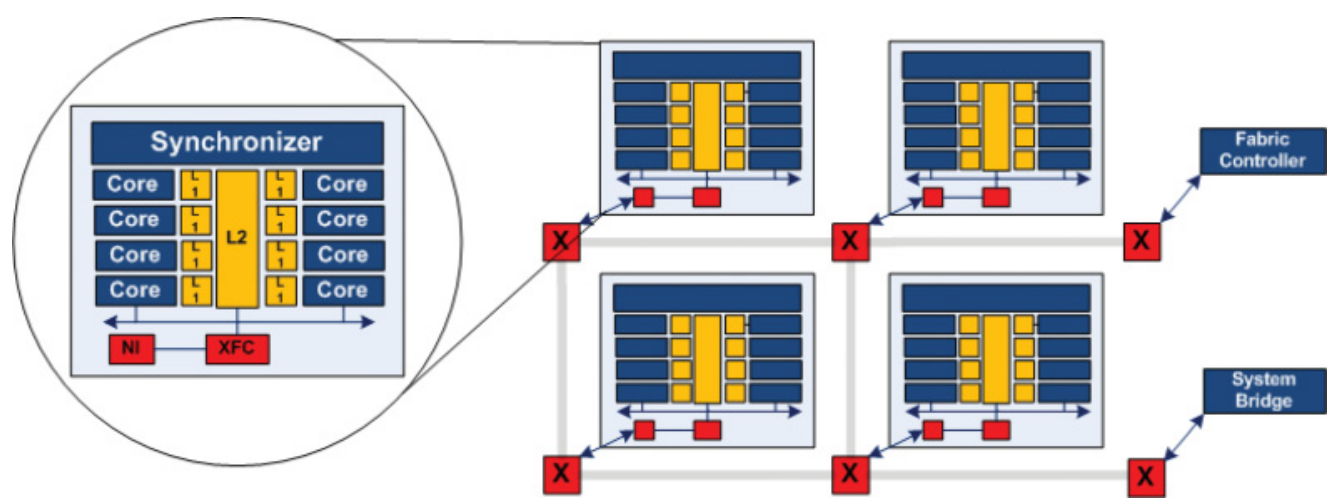

Fig. 3. Block diagram of P2012 architecture with four clusters.

and shift operations. The interconnection network consists of horizontal and vertical buses that carry data and event packets. Vertically, each object can be connected to one or more horizontal buses. In the horizontal direction, configurable Switch objects are used for segmenting the communication buses between neighboring PAEs. A configuration manager with integrated cache memory configures the array and controls the configuration sequencing.

The XPP array can be programmed in either C language or Native Mapping Language (NML), which is a low-level language for describing the computations as well as the interconnection between the processing elements.

\subsection{ST-P2012}

P2012 [P20 2010] from ST-Microelectronics, also called STHorm, is based on multiple clusters as shown in Figure 3. Up to 32 clusters are connected via a high-performance fully asynchronous network-on-chip (NoC). The current P2012 cluster is composed of a 
Table I. Summary of Distinguishing Architectural Characteristics of the Target Architectures

\begin{tabular}{|c|c|c|c|}
\hline Characteristics/Architectures & Ambric & XPP & P2012 \\
\hline \hline Core Microarchitecture & RISC+DSP & ALU+Multiplier & RISC+FPU \\
& extensions & & \\
Instruction Bit-width & 32,16 & 24 & $16 / 32 / 48$ \\
Operand Bit-width & $8 / 16 / 32$ & $8 / 16 / 24$ & $8 / 16 / 32$ \\
Instruction Pipeline & 3 -stage & - & 7 -stage \\
\hline Memory Model & Distributed local & Peripheral RAM & Partitioned Global \\
& memories & Banks & address space \\
\hline Interconnection Network & Circuit-switched & Bus based & Asynchronous NoC \\
\hline Reconfigurability Support & Programmable & Reconfigurable & Programmable \\
& cores + & ALUs + Datapath & cores + \\
& Interconnect & & [Reconfigurable \\
& & & hardware] \\
\hline
\end{tabular}

cluster controller, 16 ENcore processors, and Hardware Processing Elements (HWPEs). Each ENcore processor is implemented as a 32-bit RISC load/store architecture with floating-point operation support and a seven-stage instruction pipeline. The cluster controller is responsible for starting/stopping the execution of ENcore processors and notifying the host system. The processing elements share an advanced DMA engine, a hardware synchronizer, level 1 data memories, and an individual program cache.

The Software Development Kit (SDK) of P2012 supports the Native Programming Model (NPM), which is a low-level C-based API for abstracting the resources. An NPM application is developed by using the Architecture Description Language (ADL), the Interface Description Language (IDL), and an extended C code. ADL is used to define the structure of each component, IDL to specify the component interface, and the extended $\mathrm{C}$ language for the implementation of the code that runs on the ENcore processors and the cluster controller.

\subsection{Architecture Variability}

The distinguishing architectural features of the three target architectures are outlined in Table I.

The basic processing cores of the Ambric and P2012 platforms are based on RISC architectures, where P2012 has additional support for performing floating-point operations. On the other hand, XPP is built up of ALUs and multipliers. We also see a variation in complexity of the building blocks, starting from reconfigurable ALUs in XPP to complex PEs with extended DSP instruction set in Ambric and P2012. In Ambric, the heterogeneity is represented by two different types of processing cores (i.e., SR and SRD processors), whereas the heterogeneity in XPP is with ALU PAEs and FREGs/BREGs. A fundamental difference between the example architectures lies in the internal memory arrangement. Ambric and P2012 have local memory available within each individual basic functional unit, whereas XPP has memory banks arranged externally to the functional units and accessible by all the functional units across the array.

The communication network of P2012 supports packet-oriented routing, while Ambric has circuit-switched interconnect of channels and XPP has configurable buses. The communication network of the tiled processors Ambric and P2012 gives support to communication patterns that can be predetermined at compile time.

In terms of reconfiguration support, XPP differs from the others in the sense that it supports more fine-grained partial reconfiguration at the ALU level being controlled by the on-chip configuration manager, whereas both Ambric and P2012 rely on the external host for delivery of configuration data. In the case of Ambric, the individual processing 
cores can be reprogrammed in addition to reconfiguring their interconnection network, in contrast to P2012, where only the cores can be reprogrammed.

Given the considerable diversity in the architectural features of our three chosen target architectures and their corresponding different native programming methods, it is easily understood that it is a great challenge to develop a compilation framework that can produce efficient code for them all.

\section{OCCAM-PI LANGUAGE OVERVIEW}

Occam-pi [Welch and Barnes 2005] is a programming language based on the Communicating Sequential Processes (CSPs) [Hoare 1985] and the pi-calculus [Milner et al. 1989]. It closely resembles occam language [Occ 1995] with extensions based on the picalculus for expressing reconfigurability. It enables a minimal runtime overhead and comes with constructs for expressing parallelism and reconfigurations. Occam-pi has well-defined semantics and allows the expression of concurrent computations, interprocess communication, and reconfigurations with a formal basis. The inherent nature of the occam-pi language allows the expression of computations in the form of data flow graphs that take into account the data reuse, and the compiler can then map these data flow graphs to the target architectures. The particular features of occam-pi that are exploited include (1) the explicit concurrency with built-in mechanisms for interprocess communication that leads to the direct mapping to concurrent hardware; (2) the support for expressing dynamic parallelism in the form of replicated PAR constructs, which is being utilized by the compiler to perform resource-aware compilation in accordance with the application requirements; (3) the dynamic process invocation in the form of a dynamic forking construct that is used to express sequencing of different configurations; and (4) the support for placement, which makes it possible to direct the mapping phase to colocate interdependent processes on specific hardware resources, thus optimizing the communication costs.

The primitive processes provided by occam include assignment, input process (?), and output process (!). There are also structural processes such as sequential processes (SEQ), parallel processes (PAR), WHILE, IF/ELSE, and replicated processes [Occ 1995]. In occam-pi, the SEQ and PAR constructs can be replicated. A replicated PAR can be used to instantiate a number of processes in parallel and helps in managing the multitude of parallel resources.

In occam, the data that a process can access is strictly local and can be observed and modified by the owner process only. The communication between processes uses channels and message passing, which helps to avoid interference problems. Moreover, channel type definitions have been extended to include the direction specifiers' input (?) and output (!). Thus, a variable of a channel type refers only to one end of the channel. Channels in occam-pi are first-class citizens. Channel direction specifiers are added to the type definition of a channel and not to its name. Based on the direction specification, the compiler can do static checks of the usage of the channel both in the body of the process and in the processes that communicate with it. Channel direction specifiers are also used when referring to channel variables as parameters of a process call.

Let us now take a look at an occam-pi program that computes One-Dimensional Discrete Cosine Transform (1D-DCT). The dctpar process invokes four processes, each computing DCT in four stages, which are executed in parallel, as shown in Figure 4 . The inputs to the dctpar process are passed through input channel-end in and the results are retrieved from output channel-end out. The stage1 process contains a sequential block that takes eight input values, computes them, and passes the resulting values at its output channel. 


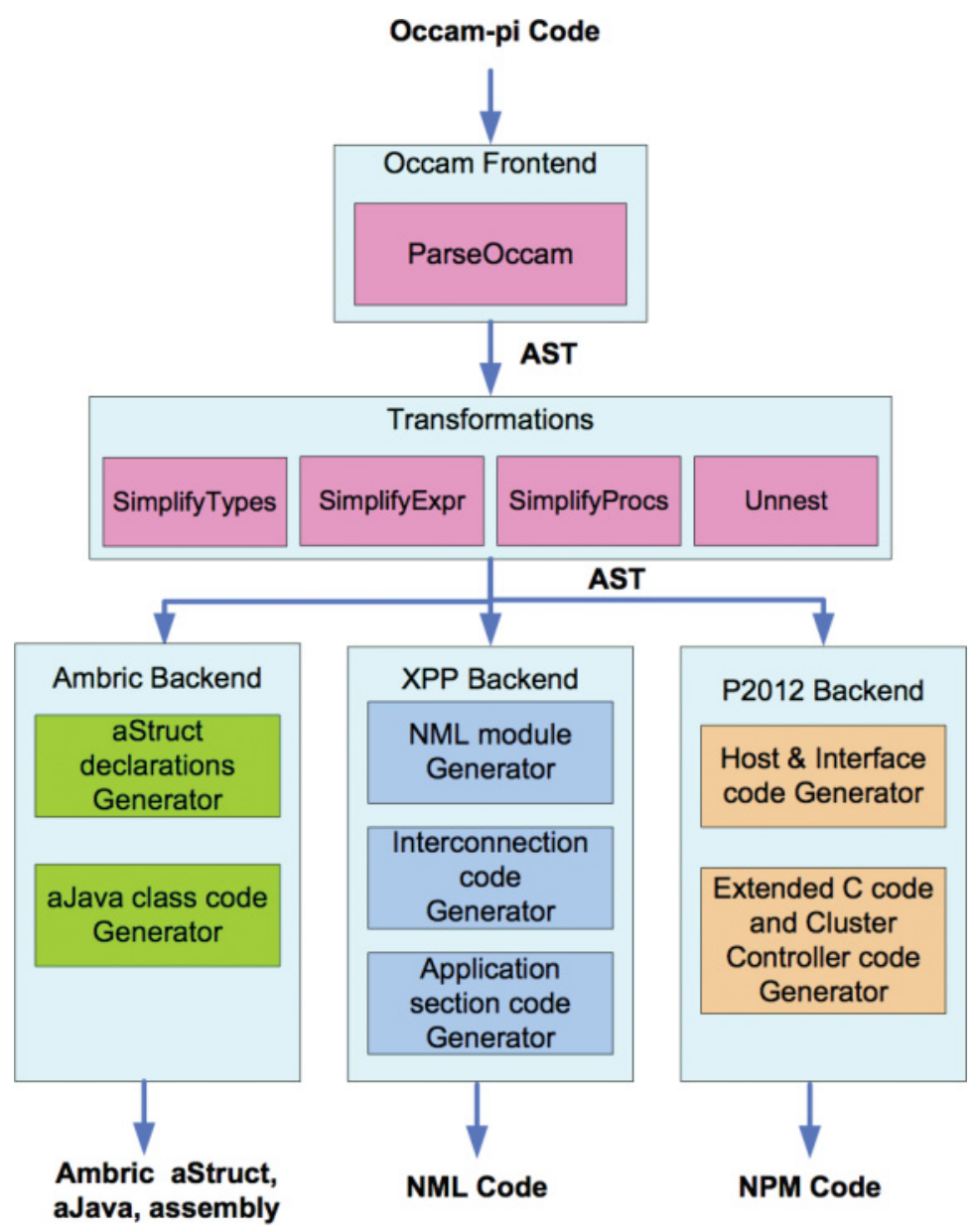

Fig. 5. Block diagram of occam-pi compiler.

integers are logical numbers that are translated by the compiler to the physical address of the resource. For a detailed description of the realization of dynamic reconfiguration of hardware resources and how the mapping of reconfigurable components is performed by making use of the placement attributes provided by occam-pi, see Ul-Abdin and Svensson [2012].

\section{COMPILER FOR RECONFIGURABLE ARRAYS}

We have adopted a source-to-source compilation approach for compiling the application specified in the occam-pi language to the native programming languages of the target architectures. We have made use of the frontend of the existing Translator from Occam to $C$ from Kent (Tock) [Tock 2008]. As shown in Figure 5, the compiler is divided into frontend, the machine-independent transformation phase, and backend, which includes the remaining target-specific phases. We have extended the frontend for supporting the language extensions of occam-pi, introduced to express reconfigurability, and developed three new backends, targeting Ambric, PACT XPP, and ST-P2012, thus generating native code in the proprietary languages aJava, assembly, aStruct, NML, and NPM. 
The key strength of our compilation methodology lies in the intermediate representation in the form of an Abstract Syntax Tree (AST) that exposes the parallelism of the program and provides abstractions to allow program portability. The modular design of the compiler framework facilitates reuse of code generation. Our compiler framework supports testing the functionality of the application by generating single-threaded C code with built-in round-robin scheduling for the concurrent processes, which can then be compiled by the GNU C compiler. The said code generator is meant for functional verification and is not suitable for performance evaluation. For the parallel execution, the generated code from the different backends can be profiled using custom tools to get the performance estimation metrics of the implementation on the target platform.

\subsection{Frontend}

The frontend of the compiler, which analyzes the occam-pi source code, consists of several modules for parsing as well as syntax and semantic analysis. The frontend tests the scope of the forking block and whether or not the data passed to a forked process is of MOBILE data type, thus fulfilling the requirement for communication semantics.

In order to support the channel end definition, we have extended the definition of channel type to include the direction whenever a channel name is found followed by a direction token, that is, "?" for input and "!" for output.

\subsection{Target-Independent Transformations}

We have devised transformations and optimizations that enable scalable implementation and at the same time produce efficient implementation. The common transformations include inlining of function calls in the processes, implementing loop unrolling, and simplifying types and expressions such as converting named data types into their underlying primitive types, resolving expression types, eliminating common subexpressions, constant folding and propagation, pulling up the SEQ replicator count, and expanding array literals.

Furthermore, we have developed partitioning and mapping techniques that favor localized communication in front of distant communication in order to minimize the impact of communication on the execution time. The employed techniques are similar to the ones used in high-level synthesis, where commonly used data is duplicated in the local memory.

\subsection{Ambric Backend}

The Ambric backend has two main passes. The first generates declarations of aStruct code, and each process in occam-pi is translated to a primitive object, which can then be executed on either an SR or an SRD processor. Before generating the aStruct code, the backend traverses the AST to collect a list of all the parameters passed in procedure calls specified for processes to be executed in parallel. This list of parameters, along with the list of names of procedures, is used to generate the structural interface and binding code for each of the parallel objects, as shown in Figure 6.

The next pass makes use of the structured composition of the occam-pi constructs, such as SEQ, PAR, and CASE, which allows intermingling processes as well as declarations and replication of constructs (like SEQ, PAR, IF). The backend uses the genStructured function to generate the aJava class code corresponding to processes that do not have the PAR construction, as shown in Figure 7. A number of optimizations such as scalarization of array variables and exploitation of instruction-level parallelism by using the mac_32_32 instruction in place of successive multiplication and addition instructions are applied in this pass. In case of the FORK construct, the backend generates the background code for managing the loading of the successive configuration from the local storage and communicating it to the concerned processing elements. 


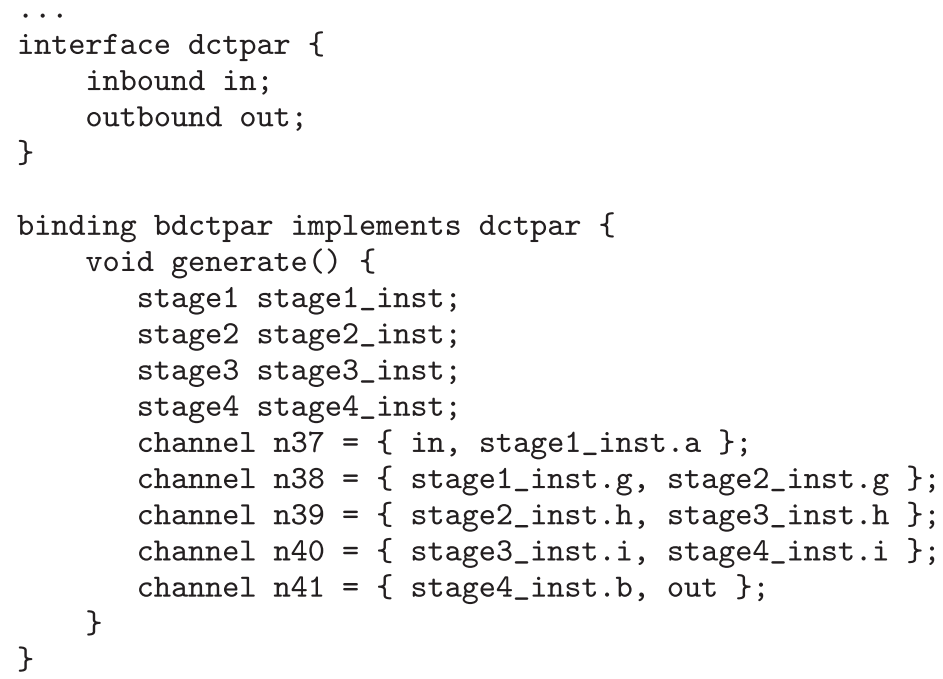

Fig. 6. Top-level aStruct code for 1D-DCT.

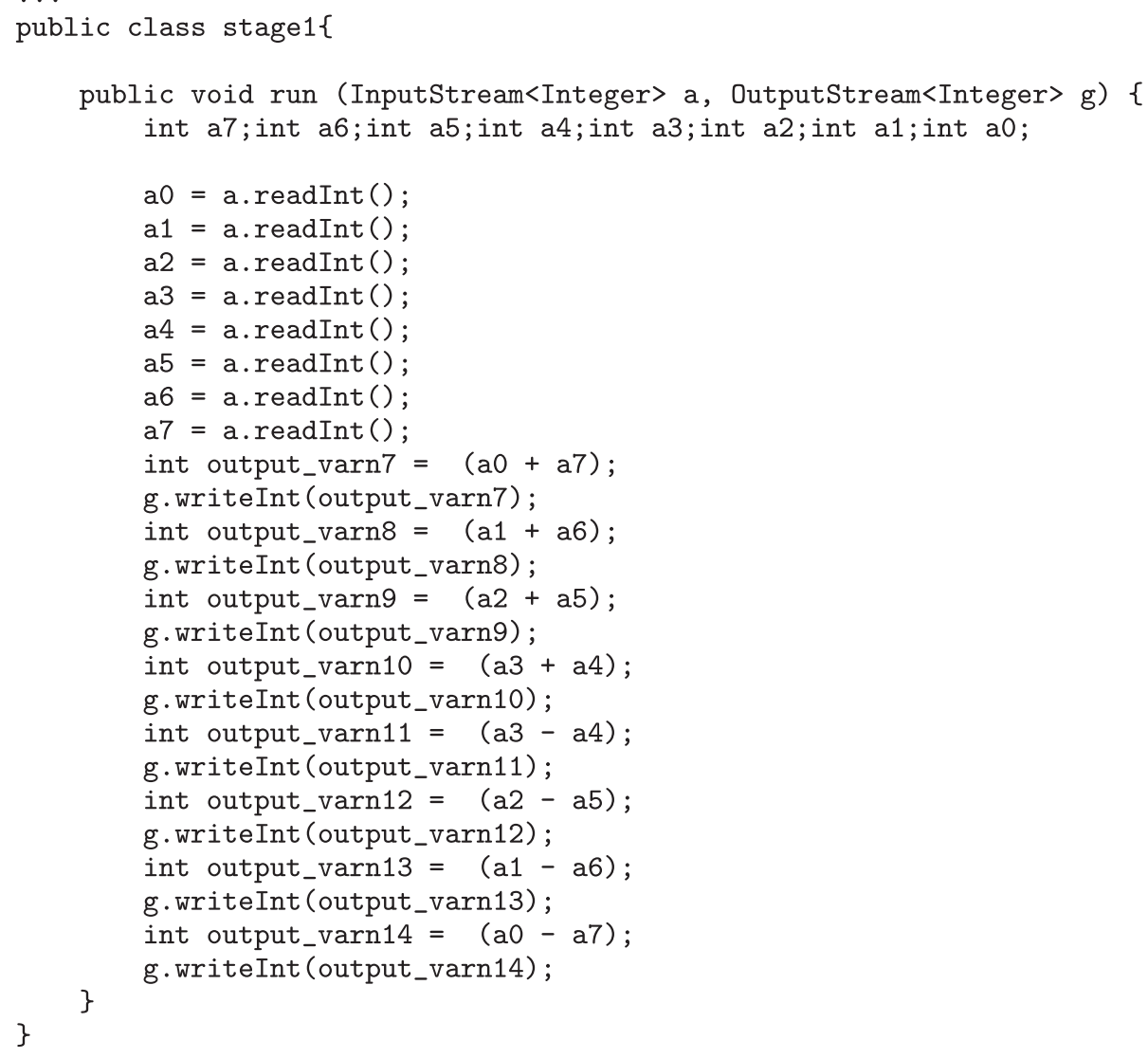

Fig. 7. aJava code for stage 1 of 1D-DCT. 


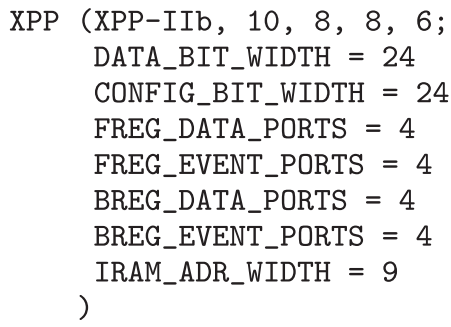

(a)

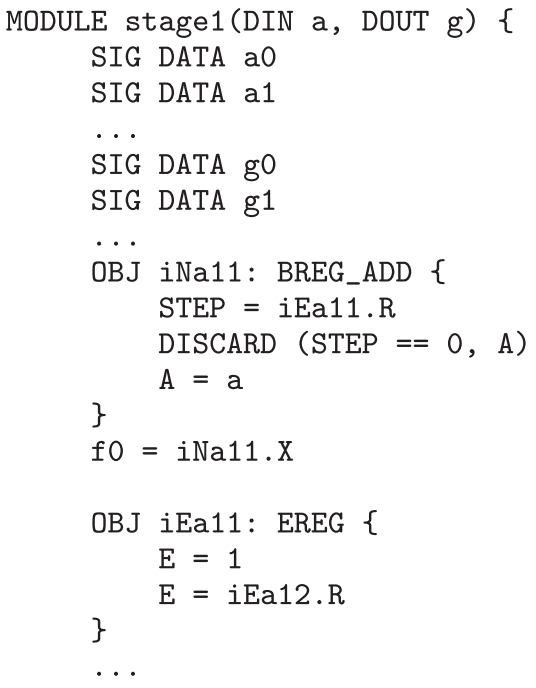

(b)

Fig. 8. NML code illustrating (a) top-level 1D-DCT and (b) parts of stage 1 implementation.

Floating-point representation is supported in the occam-pi language (in the form of REAL data types); however, it is not supported by the Ambric architecture. Thus, an automatic transformation from floating-point to fixed-point numbers has been added to this pass. Based on the dynamic range of the floating-point variables, the Q16.16 format is used to represent fixed-point numbers for the applications presented in this article. The supported arithmetic operations are as follows:

-The assignment operation converts the constant value on the right side of the operator to the selected fixed-point format. If the selected format of the left-side variable does not have enough precision for representing the constant value, then functions such as saturation, overflow, and rounding are performed on the constant.

-The add and subtract operations are applied directly without any loss of accuracy.

- The multiply operation is replaced by a function call to an assembly module.

-The division operation is also implemented as an assembly module.

\subsection{XPP Backend}

The XPP backend is divided into three main passes. The first makes use of the introduceProcSpec and genProcess functions to generate the NML module code corresponding to processes that do not have the PAR and FORK constructs. The processes invoked in a PAR construct are mapped to separate hardware resources, which operate concurrently. On the other hand, the statements enclosed within a SEQ construct are mapped to hardware resources, which operate in a pipeline manner according to the data flow patterns of the application, as shown in Figure 8(b). The backend generates a separate ALU object for each arithmetic operation, but in order to reduce the usage of ALU-PAEs, the backend can also use FREG/BREG ALUs instead of standard ALUs for implementing addition/subtraction operations, depending on the user-specified optimization flags that we have built into the compiler. An EREG object is used to synchronize the data flow between the ALU-PAEs, as seen in Figure 8(b). The replicated SEQ construct translates into a COUNT object. A COUNT object implements a counter that increments in accordance with the start and end values provided in the SEQ construct. The COUNT object 


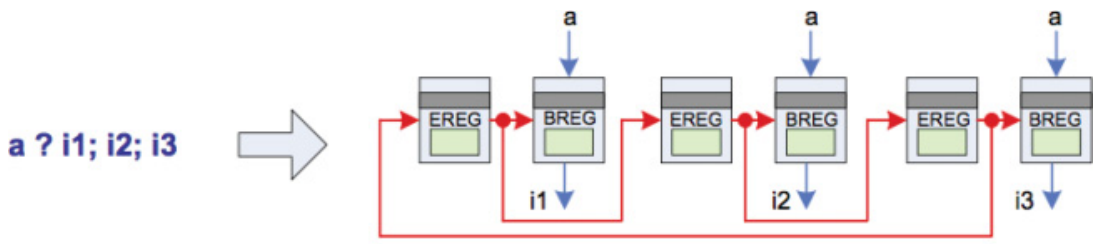

(a)

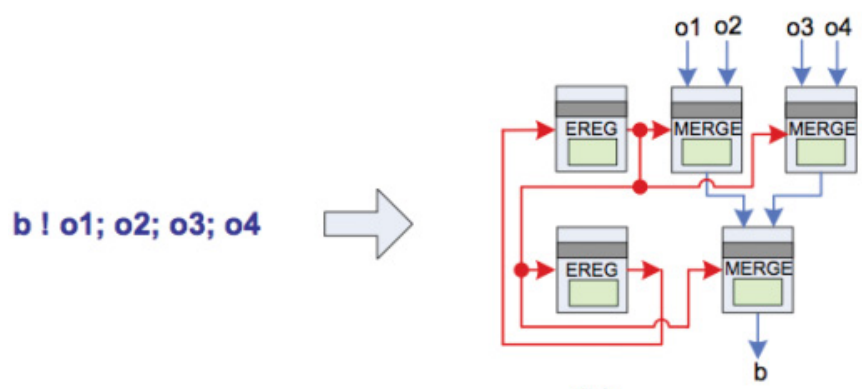

(b)

Fig. 9. Source code and resulting logic diagram illustrating (a) multiple inputs and (b) multiple outputs.

generates output packets after each increment of the counter. The array declarations are mapped to internal RAM blocks within the XPP array. Reading multiple data values from the input port and writing multiple values on the output port result in more complex control logic, as shown in Figure 9(a) and Figure 9(b), respectively.

As for Ambric, the floating-point numbers are translated into fixed-point numbers during this pass. The supported fixed-point operations are add, subtract, assign, and multiply.

The next pass generates the module definitions for the top-level process, including the interface specifications for external IOs and the interface specifications for the instantiated processes specified in the occam-pi source code, as shown in Figure 8(a). Before generating the module interface code, the backend collects a list of all the parameters passed in procedure calls specified for processes to be executed in parallel.

The final pass of the backend generates the Application section of the NML code, which is used to specify the configuration management. The Application section is generated only if the occam-pi source code of the implementation contains FORK statements. The implementation of the configuration manager, namely, configmgr process, is shown in Figure 10(a). It consists of execution of two tasks in a sequence, where task2 is forked after the completed execution of task1. The intermediate computed results of the task1 process are stored in the MOBILE data array that is mapped to the internal RAM. Figure 10(b) shows the specification of the task2 process, where its placement attributes are the same as those of the task1 process, meaning that it will be reconfigured onto the same compute resources that were previously used by the task 1 process. The translation of the logical values provided in the placement attributes to the physical addresses of the different objects within XPP is performed automatically by the compiler backend to guide the mapping process. However, in case the placement attributes are not specified in the occam-pi source, the complete mapping and routing are performed by XPP's proprietary tool.

The generated Application section consists of a number of configurations corresponding to the processes specified in the occam-pi source code, which are to be reconfigured by a forked process. The CONFIG statement describes the sequence of configurations, 


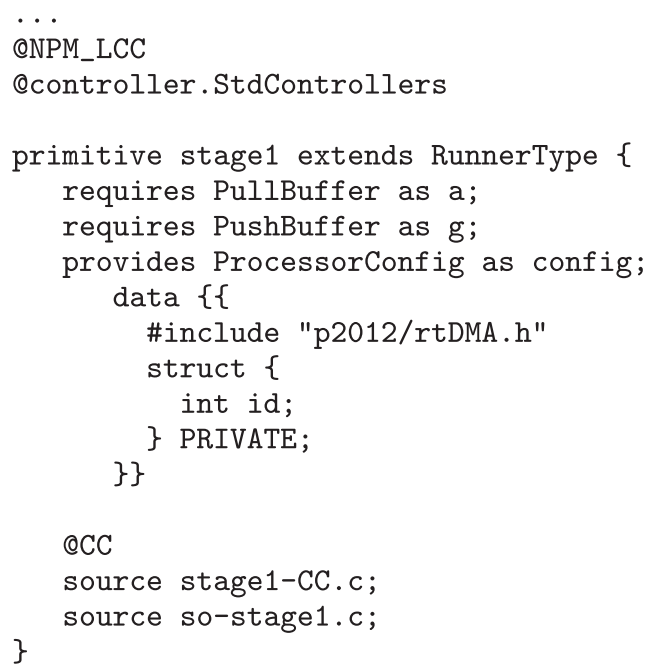

(a)

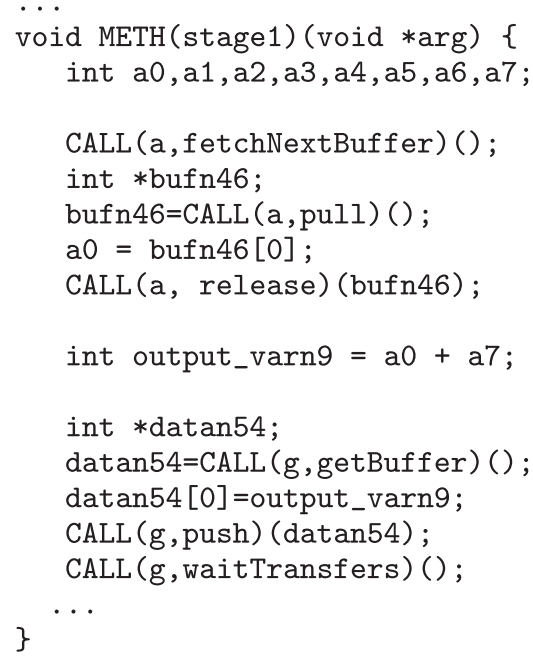

(b)

Fig. 11. NPM code for stage 1 of 1D-DCT: (a) ADL code and (b) parts of extended C code.

transformed to a list of simple processes and one parent process. This list of parameters is used to generate the required interface of each component along with its specific binding codes.

Figure 11(a) shows the architecture description code generated for a component called stage1, which corresponds to a process call in occam-pi (Figure 4). The two input and output port parameters of the stage 1 process are translated into the PullBuffer and PushBuffer services, respectively, which are provided by the NPM communication components. Since we are not using the execution engines, the cluster controller code uses runtime APIs to execute, control, and configure the application component. Cluster controller code is differentiated from the component code by inserting the @CC annotation; in Figure 11(a), the stage1-CC.c will be executed on the cluster controller, and so-stage1.c will run on the ENCore processors.

The list of parameters of process calls is also used to generate deployment, instantiation, and control code of an application component from the host side. For each process call, binary code of the process is deployed on the intended cluster using the NPMinstantiateAppComponent API, and then the cluster controller will execute this binary code on one of the ENCore processors. The NPM-instantiateFIFOBuffer API is used to bind the push buffer with the corresponding pull buffer. For replicated PAR, an array of processes is created and a for-loop is used to deploy, run, and stop the processes. The for-loop gets the start and count of the replicator from the information stored in the list of the process calls.

The second pass generates implementation code of the application components and the cluster controller and reuses the modules implemented in the Ambric backend for aJava class code generation. The genProcess function traverses the AST to generate the corresponding extended $\mathrm{C}$ code for different occam-pi primitive processes, as shown in Figure 11(b). The translation of output and input primitives of occam-pi to the corresponding NPM code is performed by using the $C A L L$ method with the push and pull parameters, respectively.

NPM components are deployed and controlled dynamically by a program running on the host system. In occam-pi, the dynamic process invocation can be used for managing the dynamic deployment of software components by the use of FORKING and FORK 
constructs. Thus, the backend will generate host-side code for a process with FORKING block. An IDL will also be generated for the interaction of an application component with the host system, and the @controller.StdControllers annotation is described at the start of the ADL file. The Comete middleware will deploy and manage the component dynamically. If the forked process makes use of MOBILE data, a communication instance will be established between the host-side program and the components to enable the transfer of data.

\section{APPLICATION CASE STUDIES}

Our compilation framework has been used quite extensively to implement fairly complex industrial applications on the three target platforms that is, XPP [Ul-Abdin and Svensson 2011], Ambric [Ul-Abdin et al. 2011; Ul-Abdin and Svensson 2012], and P2012 [Gebrewahid et al. 2013]. The applications chosen are autofocus criterion calculation for synthetic aperture radar systems and the FAST corner detection image processing algorithm. For a detailed description of these algorithms, see Ul-Abdin et al. [2011] and Gebrewahid et al. [2013], respectively. In addition, to demonstrate the applicability of our approach, we here present some common signal processing kernels applied in different application domains such as baseband processing and radar processing.

FIR Filter: An $N$-tap Finite Impulse Response (FIR) filter consists of $N$ multiplications and $N-1$ additions. We have used a streaming approach to implement the 16-tap FIR filter in occam-pi.

Matrix-Matrix Multiplication: The approach taken in matrix-matrix multiplication is to replicate one of the matrices to each process, which then computes the different elements of the resulting matrix by receiving the row-wise elements of the input matrix and multiplying them with the columns in the local copy of the other matrix.

$1 D$-DCT: 1D-DCT is used in MPEG coding to transform an $N \times N$ image block from the spatial domain to the DCT domain.

The occam-pi implementation is based on a set of filters that operate in four stages in a pipelined manner. When computing the forward DCT, a 12-bit signed vector is applied to the input on the left, and the forward DCT vector is received at the output on the right.

Reconfigurable 1D-DCT: The reconfigurable version of the 1D-DCT algorithm uses the streaming approach to conserve the hardware resources, where the four stages of computational tasks are implemented as separate configurations that can then be reconfigured on the processing resources successively. Each stage takes input values from its previous stage, performs the computation, and supplies the result to the next stage. The first and the last stages are connected to the external IOs. The implementation use FORKING and FORK constructs of occam-pi to express which tasks are to be reconfigured, and the MOBILE data type is used for the variables corresponding to the input and output values.

\section{EXPERIMENTAL EVALUATION}

We now report the experimental results of the implementations in terms of the achieved throughput using the code produced by the compiler framework and compare it with the performance achieved with manually optimized implementations. The main purpose of the evaluation is to analyze how, starting from the same application description, we can generate efficient code for a set of architectures. We also estimate the effort required to extend the compiler framework to target another architecture.

In previous implementations of complex applications, we have demonstrated the feasibility of the approach by programming complex industrial case studies in occam-pi language, viz. autofocus criterion calculation for synthetic aperture radar systems on Ambric and real-time implementation of the FAST corner detection image processing 
Table II. Utilized Resources and Performance Results

\begin{tabular}{|c|c|c|c|c|}
\hline \multirow[b]{2}{*}{$\begin{array}{l}\text { Implementations } \\
\text { in Occam-pi }\end{array}$} & \multirow[b]{2}{*}{ Target Platforms } & \multirow[b]{2}{*}{ Resources } & \multicolumn{2}{|c|}{ Performance Measure } \\
\hline & & & $\begin{array}{c}\text { Configuration } \\
\text { Overhead } \\
\text { (millisec.) }\end{array}$ & $\begin{array}{l}\text { Throughput } \\
\text { (million } \\
\text { samples/sec.) }\end{array}$ \\
\hline $\begin{array}{l}\text { 16-Tap Integer } \\
\text { FIR }\end{array}$ & $\begin{array}{r}\text { XPP@64MHz } \\
\text { Ambric@300MHz } \\
\text { P2012@600MHz }\end{array}$ & $\begin{array}{c}16 \text { ALUs } \\
16 \text { SRDs } \\
16 \text { ENcore-PEs }\end{array}$ & $\begin{array}{c}0.008 \\
0.006 \\
49\end{array}$ & $\begin{array}{c}64 \\
82.7 \\
2\end{array}$ \\
\hline $\begin{array}{l}\text { 16x16 Matrix-Matrix } \\
\text { Multiplication }\end{array}$ & $\begin{array}{r}\text { XPP@64MHz } \\
\text { Ambric@300MHz } \\
\text { P2012@600MHz }\end{array}$ & $\begin{array}{c}16 \text { ALUs } \\
4 \text { SRDs } \\
7 \text { ENcore-PEs }\end{array}$ & $\begin{array}{c}0.012 \\
0.004 \\
66\end{array}$ & $\begin{array}{c}23 \\
9.7 \\
1.34\end{array}$ \\
\hline $\begin{array}{l}1 \mathrm{D}-\mathrm{DCT} \\
8 \mathrm{x} 8\end{array}$ & $\begin{array}{r}\text { XPP@64MHz } \\
\text { Ambric@300MHz } \\
\text { P2012@600MHz }\end{array}$ & $\begin{array}{c}42 \text { ALUs } \\
4 \text { SRDs } \\
4 \text { ENcore-PEs }\end{array}$ & $\begin{array}{c}0.015 \\
0.004 \\
64\end{array}$ & $\begin{array}{c}21.3 \\
14.4 \\
0.8\end{array}$ \\
\hline $\begin{array}{l}\text { Reconfigurable } \\
\text { 1D-DCT }\end{array}$ & $\begin{array}{r}\text { XPP@64MHz } \\
\text { Ambric@300MHz } \\
\text { P2012@600MHz }\end{array}$ & $\begin{array}{c}14 \text { ALUs } \\
2 \text { SRDs } \\
2 \text { ENcore-PEs }\end{array}$ & $\begin{array}{c}0.008 \\
0.005 \\
64 \\
\end{array}$ & $\begin{array}{c}5.32 \\
7.15 \\
8 \\
\end{array}$ \\
\hline $\begin{array}{c}\text { Autofocus Criterion } \\
\text { Calculation }(1024 \times 2000)\end{array}$ & $\begin{array}{r}\text { Ambric@300MHz } \\
\text { P2012@600MHz }\end{array}$ & $\begin{array}{c}141 \text { SRDs } \\
16 \text { ENcore-PEs }\end{array}$ & $\begin{array}{c}0.043 \\
76\end{array}$ & $\begin{array}{c}0.486 \\
0.2\end{array}$ \\
\hline $\begin{array}{c}\text { FAST Corner } \\
\text { Detection }(640 \times 480)\end{array}$ & $\begin{array}{r}\text { Ambric@300MHz } \\
\text { P2012@600MHz }\end{array}$ & $\begin{array}{c}128 \text { SRDs } \\
16 \text { ENcore-PEs }\end{array}$ & $\begin{array}{c}0.035 \\
47.5 \\
\end{array}$ & $\begin{array}{c}26.8 \\
9.6\end{array}$ \\
\hline
\end{tabular}

algorithm on P2012. We have shown that this style of programming actually enables efficient programming of parallel applications, which results in competitive throughput as compared with the manual implementations. This is witnessed, for example, in the autofocus criterion calculation case study, where we were able to achieve a speedup in throughput of 11 to $23 \times$ against a state-of-the-art CPU implementation while operating at a much lower $(9 \times)$ clock frequency [Ul-Abdin et al. 2011]. In general, the occam-pi programs are much shorter, as seen in our results of the FAST corner detection algorithm, where the occam-pi program is $3 \times$ shorter, is easier to adapt, and has competitive performance when compared to programs programmed in the lowlevel native programming model of P2012 and in the high-level OpenCL language [Gebrewahid et al. 2013]. The distinguishing highlights of the occam-pi implementation include transfer of data by using arrays instead of doing it element-wise. The occam-pi implementation also benefits from avoiding the overhead of protecting shared memory accesses, since each process can only work on its own local data, and the overhead of dynamic allocation of resources by the runtime system in the hand-optimized implementation, since resource allocation is already performed at compile time.

In this work, we present the implementation results of the three kernels and the two complex case studies programmed in occam-pi and mapped to the three target architectures using the developed compiler framework. Table II presents the utilized resources in terms of number of used processing elements. The performance results are shown in terms of configuration or setup overhead, measured in used time, and throughput, measured in number of samples per second on which the given kernel can be computed. For comparison, the performance results of manually optimized implementations of the three benchmarks in the native programming languages for the three architectures are listed in Table III. The implementation results on Ambric are achieved by realizing all the designs on the GT board containing one Am2045 chip executing at $300 \mathrm{MHz}$. The results for XPP and P2012 are obtained by their respective cycle-accurate simulators considering a clock frequency of $64 \mathrm{MHz}$ and $600 \mathrm{MHz}$, respectively. For P2012, since the tools only allow us to simulate at most one cluster consisting of 16 ENcore PEs, we parameterize the benchmarks so that they do not exceed the limit of 16 cores. The I/O 
Table III. Performance Results of Manually Optimized Implementations

\begin{tabular}{|c|r|c|c|c|}
\hline Implementations & Target Platforms & $\begin{array}{c}\text { Programming } \\
\text { Languages }\end{array}$ & Resources & $\begin{array}{c}\text { Throughput } \\
\text { (million } \\
\text { samples/sec.) }\end{array}$ \\
\hline \hline & $X P P @ 64 M H z$ & NML & 16 ALUs & 64 \\
16-Tap Integer & Ambric@300MHz & aJava/aStruct & 16 SRDs & 95.6 \\
FIR & $P 2012 @ 600 M H z$ & NPM & 16 ENcore-PEs & 2.2 \\
\hline & $X P P @ 64 M H z$ & NML & 16 ALUs & 32 \\
$16 \times 16$ Matrix-Matrix & Ambric@300MHz & aJava/aStruct & 4 SRDs & 10.5 \\
Multiplication & $P 2012 @ 600 M H z$ & NPM & 4 ENcore-PEs & 2.09 \\
\hline & $X P P @ 64 M H z$ & NML & 42 ALUs & 32 \\
1D-DCT & Ambric@300MHz & aJava/aStruct & 4 SRDs & 14.8 \\
$8 \times 8$ & $P 2012 @ 600 M H z$ & NPM & 4 ENcore-PEs & 0.95 \\
\hline
\end{tabular}

data is streamed from/to the I/O channels. The input data resides in the RAM PAEs in the case of XPP, whereas it is located in the local memories of each processing element for Ambric and P2012.

The throughput results of the 16-tap FIR implementations depict that XPP can produce one output sample per cycle, whereas Ambric requires four clock cycles per output sample. The way the architectures are designed, these are the maximum throughputs that could be achieved. The utilization of resources for XPP has been optimized by using special flags for making use of ALUs available in the FREG/BREG objects for addition operations. Although the automatic generation of placement attributes of objects by the compiler backend has resulted in generating configurations that are efficient in terms of configuration overhead and latency, it could be further improved by enabling the programmer to specify the placement attributes, as is done in the hand-optimized implementation. The throughput of the P2012 implementation is much less in comparison to the other two architectures because of the overhead of the runtime system layer. This is also reflected in the increased setup time. When comparing the achieved throughput of the occam-pi FIR implementations with the hand-coded implementations, it is observed that our compiler generates competitive code with less than $13 \%$ overhead when compared to the hand-optimized code. To summarize, by using the approach of programming in high-level language and using our compiler framework, we reach competitive performance with respect to hand-optimized versions, provided, of course, that the runtime system does not result in a large overhead.

The performance results of the matrix multiplication and 1D-DCT implementations reveal a similar trend, with the XPP target producing the best throughput, followed by Ambric. The P2012 implementation again results in the least throughput, but due to the coarser granularity of computations to be performed in both of these kernels, the relative speedup is better than for the FIR implementation. This indicates that P2012 is better suited for coarse-grained parallelism than for fine-grained arithmetic operations in parallel. The difference between the utilized resources for the three targets is significant, even though we are using the same application source code. For XPP, the compiler maps the arithmetic operations defined in the sequential processes to spatially distributed hardware resources, which are then interconnected to exploit pipeline parallelism. On the other hand, the Ambric and P2012 backends map each process on an individual processing element that executes the computations imperatively and rely on instruction-level parallelism to improve performance. The comparison with the hand-optimized implementations of matrix multiplication and 1D-DCT depicts that our compiler produces comparable results in terms of the efficiency of the generated code, with some exceptions. The manually implemented matrix multiplication on XPP 
uses a custom polytope mapping model for loop parallelization [Hannig et al. 2004], which produces $35 \%$ better throughput when compared to our approach. Similarly, the matrix multiplication manually implemented on P2012 uses the host processor for distributing the input data and collecting the results, thus requiring fewer Encore-PEs and producing better throughput because of the host processor's efficiency. The occampi matrix multiplication implementation can scale for bigger matrix sizes depending on the available memory and compute resources in the target architectures. For example, Ambric and P2012 can compute up to a matrix size of $256 \times 256$ and $4096 \times 4096$, respectively, due to limited memory with each processor, whereas XPP can compute up to the matrix size of $64 \times 64$ due to limited compute resources.

In the case of the reconfigurable version of the 1D-DCT algorithm, for XPP, all the four stages of the DCT are implemented as separate configurations, which are then loaded successively. For Ambric and P2012, two pairs of processors, each performing two stages of the DCT, are reconfigured successively. The reconfiguration process is undertaken automatically and is controlled by the dedicated configuration controller and cluster controller in the case of XPP and P2012, respectively. However, for Ambric, due to the absence of the configuration controller, we used the two additional SRD processors to perform the task of configuration management. Looking at the performance results, the XPP results depict that we were able to perform reconfiguration with only a minor effect on the throughput while drastically reducing the amount of used resources (from 42 to 14 ALUs). For Ambric, the reconfiguration process is controlled in such a way that the time taken by the two processors to update their instruction memories is alternating, meaning that the first processor will be performing computations while the second one is being reconfigured. In the reconfigurable implementation using two SRD processors, most of the communication stalls that appeared in the four-processor implementation are eliminated, and time is instead used for the reconfiguration management. It is also evident from the results that the reconfiguration time is negligible in relation to the overall time of computation, indicating a reasonable feasibility of the approach.

The efficiency of the approach, in terms of the ability to use a large number of processors and specialized hardware features, is further demonstrated by the earlier performed implementations of more complex applications [Ul-Abdin et al. 2011; Gebrewahid et al. 2013]. For the autofocus criterion calculation, we are able to use a much larger number of processors (see Table II), as specified by the input parameter in the replicated PAR construct. We have implemented a number of optimizations in the Ambric backend, such as generating the assembly code for the fixed-point arithmetic used in the cubic interpolation kernel, scalarization of array variables, and exploitation of instruction-level parallelism by using mac_32_32 instruction in place of successive multiplication and addition instructions. On the other hand, we note that P2012 is able to execute the cubic interpolation kernel, which is the most demanding part of the autofocus application, much faster than Ambric, since the floating-point operations such as multiplication and division are supported by the hardware and executed in fewer cycles. Also, the FAST corner detection, based on integer arithmetic, was implemented in a much more parallelized version on Ambric than on P2012 (using 8x as many processors). This resulted in a 2.8× higher performance on Ambric than on P2012.

From the programmability point of view, it is observed that the simplicity, static compilation properties, and minimal runtime overhead of the occam-pi language, along with its power to express parallelism, helps in implementing the task of parallel programming. Programming in occam-pi exposes the communication requirements in an application in a more profound way compared to programming in imperative languages. Knowing the communication dependencies, the programmer can control the level of parallelism of the design according to the available resources and performance requirements. The existence of the REAL data type in occam-pi and the introduced conversion 
of the floating-point arithmetics to fixed point by the compiler backend also reduce the overall burden on the programmer, compared to manually implementing the fixed-point arithmetics. Furthermore, the support for expressing dynamic parallelism in the form of replicated PAR constructs enables the compiler to perform resource-aware compilation in accordance with the application requirements. The reconfigurability support allows effective reuse of resources, and the placement attributes allow processes to be colocated, which gives the potential to avoid unnecessarily expensive communication. We have experienced that the turnaround time for implementing various designs using occam-pi is significantly shorter than when using the low-level native languages of the target architectures.

When we have extended the compiler framework to target new architectures, we have started from the transformed intermediate representation in the form of the AST generated after the target-independent transformation stage. For porting to a new hardware, we can make use of the structured code generation module developed in the existing backends and tailor it for new target-specific code generation. By this reuse of the already developed infrastructure, the development effort required to extend this modular compiler framework to a new target architecture gets reduced, to less than three person-months, whereas it takes at least one person-year to develop it without reusing the existing infrastructure, all according to our experience. We would like to mention here that, currently, most architectural characteristics are hard-coded in their respective backends. One way to avoid doing so is to use an architecture description language to describe the salient features of the target architecture and provide this description to the compiler as an input. This will allow us to move some of the transformations to the target-independent phase, which would simplify the backends.

\section{CONCLUDING REMARKS}

An approach of compiling a CSP-based language to the emerging class of heterogeneous reconfigurable processor arrays has been presented. The ideas are demonstrated by a working compiler, which compiles occam-pi programs to native machine code for XPP, Ambric, and P2012 platforms. The three architectures considered in this architecture are similar in the sense that they contain many processing elements communicating over an on-chip interconnection network, yet they are different enough to allow the conclusion that the presented framework has a wider applicability. The approach has been evaluated by implementing complex industrial case studies and three common signal processing algorithms. Our proposed methodology of testing the functionality of the application in occam-pi before generating the native code reduces the turnaround time for implementing various design alternatives quite significantly, and the modularity of our compiler infrastructure enables porting to a new platform with less effort. The success of the approach stems from the well-defined semantics of the occam-pi language that allows the expression of concurrent computations, inter-process communication, and reconfigurations with a formal basis. By simplifying these tasks, the problem of efficiently mapping applications to heterogeneous reconfigurable architectures is more readily addressed, as demonstrated in this work.

\section{ACKNOWLEDGMENTS}

The authors would like to thank Nethra Imaging Inc., PACT, and ST-Microelectronics for giving access to their software development suites and hardware boards.

\section{REFERENCES}

1995. Occam ${ }^{\circledR} 2.1$ Reference Manual. SGS-Thomson Microelectronics Limited.

2002. Handel-C Language Reference Manual, Version 3.1. Celoxica Inc. 
2006. Mobius Language Manual. Codetronix LLC.

2010. Platform 2012: A Manycore Programmable Accelerator for Ultra-Efficient Embedded Computing in Nanometer Technology. Technical Report. ST-Microelectronics and CEA.

Fred Barnes and Peter H. Welch. 2002. Prioritised dynamic communicating processes - Part II. In Communicating Process Architectures 2002. 353-370.

Volker Baumgarte, G. Ehlers, Frank May, Armin Nuckel, Martin Vorback, and Markus Weinhardt. 2003. PACT XPP: A self-reconfigurable data processing architecture. Journal of Supercomputing 26, 2 (2003), 167-184. DOI : http://dx.doi.org/10.1023/A:1024499601571

Srinivas Boppu, Frank Hannig, and Jürgen Teich. 2014. Compact code generation for tightly-coupled processor arrays. Journal of Signal Processing Systems 77, 1-2 (2014), 5-29. DOI:http://dx.doi.org/ 10.1007/s11265-014-0891-2

Jim Burns, Adam Donlin, Jonathan Hogg, Satnam Singh, and Mark de Wit. 1997. A dynamic reconfiguration run-time system. In Proceedings of the 5th International Symposium on Field-Programmable Custom Computing Machines (FCCM'97).

João M. P. Cardoso, Pedro C. Diniz, and Markus Weinhardt. 2010. Compiling for reconfigurable computing: A survey. ACM Computing Survey 42, 4, Article 13 (June 2010), 65 pages. D0I:http://dx.doi.org/ $10.1145 / 1749603.1749604$

Joao M. P. Cardoso and Markus Weinhardt. 2002. XPP-VC: A C compiler with temporal partitioning for the PACT-XPP architecture. In Proceedings of the 12th International Conference on Field Programmable Logic and Applications (FPL'02). Springer-Verlag, 864-874.

Jan Frigo, Maya Gokhale, and Dominique Lavenier. 2001. Evaluation of the streams-C C-to-FPGA compiler: An applications perspective. In Proceedings of the 9th International Symposium on Field-Programmable Gate Arrays (FPGA'01). ACM, 134-140. DOI:http://dx.doi.org/10.1145/360276.360326

Essayas Gebrewahid, Zain Ul-Abdin, Bertil Svensson, Veronica Gaspes, Bruno Jego, Bruno Lavigueur, and Mathieu Robart. 2013. Programming real-time image processing for manycores in a high-level language. In Proceedings of the Advanced Parallel Processing Technologies. Lecture Notes in Computer Science, Vol. 8299. Springer, Berlin, 381-395. DOI : http://dx.doi.org/10.1007/978-3-642-45293-2_29

Michael I. Gordon, William Thies, Michal Karczmarek, Jasper Lin, Ali S. Meli, Andrew A. Lamb, Chris Leger, Jeremy Wong, Henry Hoffmann, David Maze, and Saman Amarasinghe. 2002. A stream compiler for communication-exposed architectures. SIGARCH Computer Architecture News 30, 5 (2002), 13. DOI : http://dx.doi.org/10.1145/635506.605428

David Greaves and Satnam Singh. 2008. Kiwi: Synthesis of FPGA circuits from parallel programs. In Proceedings of the International Symposium on Field-Programmable Custom Computing Machines (FCCM'08). http://research.microsoft.com/apps/pubs/default.aspx?id=71425.

Frank Hannig, Hritam Dutta, and Jrgen Teich. 2004. Mapping of regular nested loop programs to coarsegrained reconfigurable arrays - Constraints and methodology. In Proceedings of the 18th Parallel and Distributed Processing Symposium (IPDPS'04).

C. A. R. Hoare. 1985. Communicating Sequential Processes. Prentice-Hall.

Anthony Mark Jones and Michael Butts. 2006. TeraOPS hardware: A new massively-parallel MIMD computing fabric IC. In Proceedings of IEEE Hot Chips Symposium.

Jong-eun Lee, Kiyoung Choi, and Nikil D. Dutt. 2003. Compilation approach for coarse-grained reconfigurable architectures. IEEE Design and Test of Computers 20, 1 (2003), 26-33. DOI:http://dx.doi.org/ 10.1109/MDT.2003.1173050

Wayne Luk and Steve Mckeever. 1998. Pebble: A language for parametrised and reconfigurable hardware design. In Field-Programmable Logic and Applications From FPGAs to Computing Paradigm. Springer, 9-18. DOI: http://dx.doi.org/10.1007/BFb0055228

Bingfeng Mei, S. Vernalde, D. Verkest, H. De Man, and R. Lauwereins. 2002. DRESC: A retargetable compiler for coarse-grained reconfigurable architectures. In Proceedings of the International Conference on FieldProgrammable Technology (FPT'02). 166-173. DOI : http://dx.doi.org/10.1109/FPT.2002.1188678

Robin Milner, Joachim Parrow, and David Walker. 1989. A calculus of mobile processes, Part I. Information and Computation 100, 1 (1989), 1-40.

Alexandros Papkonstantinou, Yun Liang, John A. Stratton, Karthik Gururaj, Deming Chen, Wen-Mei W. Hwu, and Jason Cong. 2011. Multilevel granularity parallelism synthesis on FPGAs. In Proceedings of the International Symposium on Field-Programmable Custom Computing Machines (FCCM'11). DOI : http://dx.doi.org/10.1109/FCCM.2011.29

Hyunchul Park, Yongjun Park, and Scott Mahlke. 2009. Polymorphic pipeline array: A flexible multicore accelerator with virtualized execution for mobile multimedia applications. In Proceedings of the 42nd Annual IEEE/ACM International Symposium on Microarchitecture (MICRO 42). 11. DOI : http://dx.doi.org/10.1145/1669112.1669160 
Tock. 2008. Tock: Translator from Occam to C by Kent. http://projects.cs.kent.ac.uk/projects/tock/trac/.

Zain Ul-Abdin, Anders Ahlander, and Bertil Svensson. 2011. Programming real-time autofocus on a massively parallel reconfigurable architecture using occam-pi. Proceedings of the International Symposium on Field-Programmable Custom Computing Machines (FCCM'11), 194-201. DOI :http://dx.doi.org/ 10.1109/FCCM.2011.20

Zain Ul-Abdin, E. Gebrewahid, and B. Svensson. 2012. Managing dynamic reconfiguration for fault-tolerance on a manycore architecture. In Proceedings of the 26th International Symposium on Parallel and Distributed Processing Workshops (IPDPSW'12). 312-319. DOI : http://dx.doi.org/10.1109/IPDPSW.2012.38

Zain Ul-Abdin and B. Svensson. 2011. Occam-pi as a high-level language for coarse-grained reconfigurable architectures. In Proceedings of the 25th International Symposium on Parallel and Distributed Processing Workshops (IPDPSW'11). 236-243. DOI : http://dx.doi.org/10.1109/IPDPS.2011.147

Zain Ul-Abdin and B. Svensson. 2012. Occam-pi for programming of massively parallel reconfigurable architectures. International Journal of Reconfigurable Computing 2012 (2012), Article No. 1. DOI : http://dx.doi.org/ doi:10.1155/2012/504815

Peter H. Welch and Frederick R. M. Barnes. 2005. Communicating mobile processes: Introducing occam-pi. In 25 Years of CSP (Lecture Notes in Computer Science), Vol. 3525. Springer Verlag, 175-210. http://www.cs. kent.ac.uk/pubs/2005/2162

Michael J. Wirthlin and Brad L. Hutchings. 1995. A dynamic instruction set computer. In Proceedings of IEEE Workshop on FPGAs for Custom Computing Machines. 99-107.

Received November 2014; revised September 2015; accepted November 2015 